\title{
Simulação Realística como Estratérgia Facilitadora no Ensino- aprendizagem para Identificação dos Riscos no Paciente Cirúrgico Oncológico
}

\author{
Realistic Simulation as a Facilitating Strategy in Teaching-Learning for Risk Identification in Cancer \\ Surgical Patients \\ Simulación realista como estrategia facilitadora en la enseñanza-aprendizaje para la identificación \\ de riesgos en pacientes quirúrgicos de cáncer
}

\section{Lilia Dias Santana de Almeida Pedrada ${ }^{*}$, Ana Karine Ramos Brum² \\ Como citar esse artigo. Pedrada, LDSA;}

Brum, AKR. Simulação Realística como

Estratérgia Facilitadora no Ensinoaprendizagem para Identificação dos Riscos no Paciente Cirúrgico Oncológico. Revista Pró-UniverSUS. 2020 Jul./Dez.; 11 (2): 195-198.

\author{
Resumo
}

Esse estudo traz como objeto à utilização da simulação realística como tecnologia educacional visando uma estratégia facilitadora do ensino na gestão do cuidado seguro através da visita pré-operatória de enfermagem. Objetiva validar a estratégia da simulação realística aplicada no ensino da visita pré-operatória para residência de enfermagem oncológica, visando identificar os riscos no processo da gestão do cuidado seguro. A proposta metodológica da pesquisa será realizada em caráter descritivo e exploratório, com abordagem qualitativa de desenvolvimento metodológico, a qual caracteriza-se pela validação de tecnologias educacionais, o cenário de atuação da simulação realística se dará na unidade II no Instituto Nacional do Câncer (INCA). Como instrumentos de coleta de dados serão utilizados questionário pré e pós teste (para os participantes), questionário de validação (para os juízes especialistas), habilitação de curso referência em simulação realística, atores e/ou manequins e roda de conversa (debriefing), os participantes da pesquisa serão os discentes de enfermagem do Programa de Residência Multiprofissional Oncológica do Instituto Nacional do Câncer (INCA), eles deverão assinar o termo de consentimento livre e esclarecido. E como resultado, acredita se que esse estudo contribua com a formação do residente de enfermagem sob o olhar da gestão do cuidado seguro através da visita pré operatória e pretende-se produzir como produto um treinamento aplicando a simulação realística como metodologia ativa. Acredita-se que com o processo ensino aprendizagem (enfermeiro preceptor e residente de enfermagem), onde podemos destacar a ação participativa no fazer pedagógico através da metodologia ativa, a formação profissional se dará com ênfase na segurança do paciente cirúrgico.

Palavras-chave: Segurança do Paciente, Simulação Realística, Residência em enfermagem, Visita Pré-operatória.

\begin{abstract}
This study has as its object the use of realistic simulation as educational technology aiming at a facilitating strategy of teaching in the management of safe care through the preoperative nursing visit. It aims to validate the strategy of realistic simulation applied in the teaching of the preoperative visit to the oncology nursing residence, aiming to identify the risks in the safe care management process. The methodological proposal of the research will be carried out in a descriptive and exploratory character, with a qualitative approach of methodological development, which is characterized by the validation of educational technologies, the scenario of performance of realistic simulation will take place in unit II at the National Cancer Institute (INCA). ). As data collection tools will be used pre and post test questionnaire (for participants), validation questionnaire (for expert judges), enabling realistic reference simulation course, actors and / or dummies and debriefing, The research participants will be the nursing students of the National Cancer Institute (INCA) Multiprofessional Oncology Residency Program, and they must sign the free and informed consent form. And as a result, it is believed that this study contributes to the education of nursing residents from the perspective of safe care management through the preoperative visit and is intended to produce as a product training by applying realistic simulation as an active methodology. It is believed that with the teaching-learning process (preceptor nurse and nursing resident), where we can highlight the participatory action in the pedagogical practice through the active methodology, the professional training will take place with emphasis on surgical patient safety.
\end{abstract}

Keywords: Patient Safety, Realistic simulation, Nursing residence, Preoperative visit.

Afiliação dos autores:

${ }^{1}$ Enfermeira. Mestranda do programa de Mestrado Profissional em Ensino na Saúde/MPES, Universidade Federal Fluminense (UFF). Niterói, RJ. Brasil. Email: liliapedrada@ hotmail.com OR $\neg$ CID: https://orcid.org/0000-0001-5692-0699

${ }^{2}$ Enfermeira. Pós-Doutora em Enfermagem. Professora do Programa de Mestrado Profissional em Ensino na Saúde/MPES, Universidade Federal Fluminense (UFF). Programa de Mestrado Profissional em Ensino na Saúde/MPES, UFF, RJ, Brasil. Email: karinebrum@yahoo.com.br ORCID: https://orcid.org/0000-0002-1071-3587 


\section{Resumen}

Este estudio tiene como objetivo el uso de simulación realista como tecnología educativa con el objetivo de facilitar la estrategia de enseñanza en el manejo de la atención segura a través de la visita de enfermería preoperatoria. Su objetivo es validar la estrategia de simulación realista aplicada en la enseñanza de la visita preoperatoria a la residencia de enfermería oncológica, con el objetivo de identificar los riesgos en el proceso de gestión de la atención segura. La propuesta metodológica de la investigación se llevará a cabo en un carácter descriptivo y exploratorio, con un enfoque cualitativo de desarrollo metodológico, que se caracteriza por la validación de las tecnologías educativas, el escenario de ejecución de simulación realista tendrá lugar en la unidad II del Instituto Nacional del Cáncer (INCA). ) Como las herramientas de recolección de datos se utilizarán antes y después del cuestionario de prueba (para los participantes), cuestionario de validación (para jueces expertos), permitiendo un curso de simulación de referencia realista, actores y / o maniquíes y sesiones informativas, Los participantes de la investigación serán los estudiantes de enfermería del Programa de Residencia de Oncología Multiprofesional del Instituto Nacional del Cáncer (INCA), y deben firmar el formulario de consentimiento libre e informado. Y como resultado, se cree que este estudio contribuye a la educación de los residentes de enfermería desde la perspectiva de la gestión segura de la atención a través de la visita preoperatoria y está destinado a producir como un producto de capacitación mediante la aplicación de simulación realista como metodología activa. Se cree que con el proceso de enseñanza-aprendizaje (enfermera preceptora y residente de enfermería), donde podemos destacar la acción participativa en la práctica pedagógica a través de la metodología activa, la capacitación profesional se llevará a cabo con énfasis en la seguridad del paciente quirúrgico.

Palabras clave: : Seguridad del paciente, Simulación realista, Residencia de enfermería, Visita preoperatoria.

\section{Introdução}

Com uma trajetória de mais de 20 anos em centro cirúrgico, pude observar o quanto o paciente cirúrgico é vulnerável à riscos e danos no período transoperatório, em virtude de um ambiente altamente dinâmico e tecnológico. O Centro cirúrgico é o local onde são realizados procedimentos cirúrgico-anestésicos. É um cenário com uma dinâmica particular, onde a pressão pelo tempo, resultados e práticas complexas e interdisciplinares marcam o cotidiano. $\mathrm{O}$ estresse e aceleração do trabalho agravam este ambiente, aumentando a susceptibilidade a ocorrência de eventos adversos ${ }^{1}$.

Muitas vezes trata-se de um ambiente estressante e hostil devido a demanda de tarefas, o que estimula o silêncioe odistanciamento entre a equipe multidisciplinar e o paciente, se transformando em um local com muitos riscos ao paciente.

Penso que esses riscos são realidades presentes na assistência cirúrgica e cabe às equipes envolvidas no processo propor estratégias e estabelecer barreiras para garantir a segurança do paciente. Dentre os riscos identificados na prática são, por exemplo, risco de queda, de lesão de pele, de sangramento, de infecção, de queimadura, de reação alérgica, de lateralidade, entre outros. Desta forma é imprescindível avaliar criteriosamente todos esses riscos, com a intenção de prevenir ou reduzir a possibilidade de complicações posteriores.

A assistência sistematizada é baseada em conhecimento científico e essencial para garantir a assistência de enfermagem de qualidade e segurança para o paciente cirúrgico. Assim, julgo ser relevante destacar a Sistematização da Assistência de Enfermagem Perioperatória (SAEP) aplicada na visita pré-operatória de enfermagem como uma ferramenta para garantir a segurança do paciente. Pois durante a aplicação da SAEP é possível identificar as situações de riscos inerentes de todo ambiente que permeia o processo cirúrgico no período perioperatório e, então, planejar as ações que minimizem os riscos existentes.

A visita pré-operatória de Enfermagem é a atividade primordial do Enfermeiro no período préoperatório, pois, além de representar o primeiro contato entre Enfermeiro e paciente, possibilita ao profissional detectar, solucionar e/ou encaminhar problemas identificados. A visita pré-operatória representa a essência para o sucesso da Sistematização da Assistência de Enfemagem Perioperatória-SAEP, é a primeira etapa desse processo e somente a partir desta, é possível dar continuidade à assistência nas fases subseqüentes ${ }^{2}$.

A relação de ensino-aprendizagem ou aluno residente-preceptor, que se efetiva nessa formação prática, deveria se configurar como processo de aprendizagem significativa, no qual ao conhecimento, já experienciado pelo aluno em momentos anteriores de formação, se acresçam novos, potencializando e qualificando esse processo e, consequentemente, a assistência prestada aos usuários que demandam aos serviços ${ }^{3}$.

Acredito que o padrão ouro na pós-graduação da enfermagem é a residência, ela tem como objetivo especializar o enfermeiro para o mercado de trabalho nos moldes de treinamento em serviço, propicia qualificação acadêmica e preparo técnico-científico em área especializada.

Os cursos de especialização e aperfeiçoamento tem o objetivo técnico-profissional específico sem abranger o campo total do saber em que insere a especialidade. São cursos destinados ao treinamento nas partes de que se compõe um ramo profissional ou científico. Sendo assim, é entendido ser sua meta, o domínio científico e técnico de uma certa e limitada área do saber ou da profissão para formar o profissional especializado ${ }^{4}$.

Acreditamos que a residência em enfermagem é um momento de grande aprendizado teórico-prático, o principal objetivo é proporcionar ao enfermeiro 
residente o acesso a um conjunto de atividades/ações que articulem os conhecimentos referentes à pesquisa, à assistência, à extensão e ao ensino de enfermagem, qualificando-o como profissional crítico e inserido no debate sobre o desenvolvimento técnico-científico. Portanto julgamos ser de extrema importância a atuação do residente de enfermagem no centro cirúrgico e, em particular, no momento pré-operatório ${ }^{5}$.

Pelo fato de essa modalidade de capacitação oferecer oportunidade de crescimento, liderança, gerência e desenvolvimento de habilidades técnicas relacionadas ao cuidado de pacientes com variados níveis de complexidade, a presença do preceptor é essencial em termos de supervisão e suporte de práticas seguras $^{6}$

Dentre as estratégias da metodologia ativa, destaca-se a simulação realista, que, como estratégia da educação em enfermagem, é definida como uma técnica que utiliza tecnologias para replicar cenários que simulam a prática em um ambiente controlado e realista. Nele, o aluno participa ativamente do processo de ensino e aprendizagem para praticar exaustivamente, aprender, refletir e avaliar produtos e processos. Ao realizar cenários, o aluno se depara com uma realidade que exigirá seu conhecimento para sua resolução ${ }^{7}$.

A simulação realística é vista como um método efetivo e inovador que amplia as relações entre a teoria e a prática do corpo discente em ambiente seguro, oferecendo melhores oportunidades de aprendizagem e treinamento, contribuindo para a formação profissional. A última etapa da simulação permite uma discussão reflexiva (debriefing) sobre a situação ocorrida, da aprendizagem e das decisões tomadas, estimulando o pensamento crítico e reflexivo do estudante, consolidando os saberes. A simulação na educação dos profissionais de saúde dá aos estudantes a oportunidade de desenvolverem habilidades variadas e competências necessárias em ambiente controlado e protegido. Permite erros e crescimento profissional, sem arriscar a segurança do paciente ${ }^{8}$.

A simulação realística tem sido utilizada como recurso tecnológico no ensino em ciências da saúde, uma metodologia de ensino fundamentada na Aprendizagem Baseada em Problema (ABP), que possibilita ao estudante experiências formativas nas diversas situações clínicas baseadas em fatos reais e em ambiente seguro, na qual o erro expõe a segurança do paciente a riscos, além de possibilitar ao estudante um raciocínio clinico, desempenho prático, aquisição de habilidades e melhoria na comunicação entre a equipe multiprofissional e o paciente. $\mathrm{O}$ uso da simulação clínica no processo de ensino-aprendizagem tem sido assunto de estudos recentes e tem demonstrado ser um método eficaz no processo de ensino aprendizagem e que desperta maior interesse por parte dos discentes. Esta metodologia pode possibilitar uma formação mais robusta ao consolidar teoria e prática simultaneamente. A metodologia de simulação realística contribui ainda como instrumento pedagógico para a uma comunicação efetiva, o que resulta em uma maior qualidade da assistência e segurança do paciente, direcionando-o para uma formação de um profissional difusor de um cuidado seguro e consciente das suas ações ${ }^{9}$.

Como preceptora observo os residentes de enfermagem do programa de residência multiprofissional de um Instituto em Oncologia do Rio de Janeiro, quando submetidos ao campo prático em Centro Cirúrgico, percebo a ausência total ou parcial de conhecimento do conteúdo sobre SAEP, com ênfase na visita préoperatória de enfermagem, trazendo dificuldades para o desenvolvimento das atividades na área específica. Emerge então, minha inquietação e preocupação para utilizar a visita pré-operatória como estratégia para segurança do paciente com a visão dos residentes de enfermagem em oncologia.

\section{Objetivo}

Validara estratégia da simulaçãorealística aplicada no ensino da visita pré-operatória para residência de enfermagem oncológica, visando identificar os riscos no processo da gestão do cuidado seguro.

\section{Metodologia}

A pesquisa será realizada em caráter descritivo e exploratório, com abordagem qualitativa de desenvolvimento metodológico, a qual caracteriza-se pela validação de tecnologias educacionais.

Foram respeitados os aspectos éticos, com aprovação, parecer número: 4.342.083.

Os participantes da pesquisa serão os discentes de enfermagem do Programa de Residência Multiprofissional Oncológica do Instituto Nacional do Câncer-INCA. O cenário de atuação da simulação realística se dará na unidade II no Instituto Nacional do Câncer.

Esse processo irá se desenvolver em 6 etapas;

$1^{\text {a }}$ Etapa - Identificar o conhecimento científico dos residentes de enfermagem do programa de residência multiprofissional em oncologia através da estratégia pré teste da simulação realística sobre a temática da visita pré operatória de enfermagem.

- Instrumento: Questionário validado por juízes especialistas com abordagem sobre gestão do cuidado seguro através da visita pré-operatória de enfermagem

- Análise: Dados do questionário no pré teste respondido pelos residentes de enfermagem.

$2^{\text {a }}$ Etapa: Elaborar um roteiro de simulação e organização dos cenários para a gestão do cuidado seguro para os residentes de enfermagem do Programa 
de Residência Multiprofissional em Oncologia.

- Instrumento: Resultado do curso de habilitação específica sobre simulação realística por parte do preceptor

- Análise: Validação por juízes especialistas

$3^{\text {a }}$ Etapa; Aplicar a metodologia de Simulação Realística como ferramenta pedagógica.

- Instrumento: Laboratório com materiais e insumos para simulação realística para identificação dos riscos eminentes do processo cirúrgico (serão descritos após habilitação do preceptor em curso de referência na temática).

- Análise: Identificar resultados da metodologia ativa.

$4^{a}$ Etapa: Realizar pós teste seguido de debriefing após a simulação realística, comparando com pré teste, tornando imperativa a inclusão do residente no processo ensino/aprendisagem.

- Instrumento: Questionário e roda de conversa

- Análise: Discussão participativa preceptor/ residente sobre a estratégia facilitadora da Tecnologia Educacional.

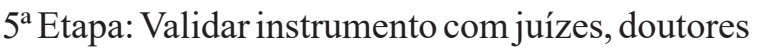
em centro cirúrgico ou de ensino, através do currículo lattes ou método bola de neve. forms)

- Instrumento: Questionário de validação (google

- Análise: Resultados do instrumento de validação por respostas diretas e contribuições discursiva pelos juízes especialistas.

$6^{a}$ Etapa: Propor um plano de inserção do produto na prática do ensino em saúde na formação profissional dos residentes de enfermagem do curso de residência multiprofissional em oncologia.

- Instrumento: Simulação Realística como estratégia facilitadora da Tecnologia Educacional.

- Análise: Desenvolvimento da ação da visita pré operatóriana gestão do cuidado seguro no campo prático do residente de enfermagem.

\section{Resultados Esperados}

E como resultado, acredita se que esse estudo contribua com a formação do residente de enfermagem sob o olhar da gestão do cuidado seguro através da visita pré operatória e pretende-se produzir como produto um treinamento aplicando a simulação realística como metodologia ativa. Onde o processo ensino aprendizagem (enfermeiro preceptor e residente de enfermagem), poderá ter destaque na ação participativa do fazer pedagógico através da Tecnologia educacional, e a formação profissional se dará com ênfase na segurança do paciente cirúrgico.

\section{Referências}

1. Ribeiro, E; Ferraz, K.M.C; Duran, E.C.M. Rev. SOBECC, São Paulo. Out/Dez. 2017; 22(4): 201-207, Atitudes dos enfermeiros de centro cirúrgico diante da Sistematização da Assistência de Enfermagem Perioperatória.

2. Oliveira, M.M; Mendonça, K.M; Análise da visita pré-operatória de enfermagem: revisão integrativa. Rev SOBECC. 2014;19(3):164-72.

3. Antunes, J. M; Daher, D. V; Ferrari, M. F. Preceptoria como lócus de aprendizagem e de coprodução de conhecimento/Preceptory as locus of learning and coproductionofknowledge.Rev.enferm. UFPE on line; 11(10): 3741-3748, out.2017.

4. Aguiar et al. Rev Bras Enferm, Brasília (DF) 2004 set/ out;57(5):555-9

5. Bosco, P. S; Santiago, L. C; Costa, A. J; Oliveira, M.S.D.R; Carneiro, B. M; Ferreira, E. F. A visita pré-operatória de enfermagem pelo residente de enfermagem médico-cirúrgica: Relato de experiência. Revista de enfermagem UFPE on line., Recife, 7(11):6553-6, nov., 2015.

6. Oliveira, E.B; Carvalho, R; Teixeira, E; Zeitoune, R, C, G; Sabóia, V. M; Gallasch, C, H. Fatores intervenientes na formação de enfermeiros residentes: visão de egressos de um programa de residência. REME - Rev Min Enferm. 2017

7. Janicas, R. C. S. V; Narch, N. Z; Evaluation of nursing students' learning using realistic scenarios with and without debriefing. Rev. LatinoAm. Enfermagem 2019; 27: e 3187.

8. Ferreira, R.P; Guedes, H. M; Oliveira, D. W. D; Simulação Realística como Estratégia de Ensino no Aprendizado de Estudantes da Área da Saúde. Revista de Enfermagem do Centro-Oeste Mineiro.2018; DOI: http://dx.doi. org/10.19175/recom.v7i0.2508

9. Rohrs, R. M. S; Santos, C. F; Barbosa, R. S; Schulz, R. S; Carval, M. B; Simulação realística e seus atributos para formação do enfermeiro. Revista de enfermagem UFPE on line., Recife, 2017. 